\title{
Advances in 3D neuronal cell culture
}

Jean-Philippe Frimat, Sijia Xie, Alex Bastiaens, Bart Schurink, Floor Wolbers, Jaap den Toonder, and Regina Luttge

Citation: Journal of Vacuum Science \& Technology B 33, 06F902 (2015); doi: 10.1116/1.4931636

View online: https://doi.org/10.1116/1.4931636

View Table of Contents: https://avs.scitation.org/toc/jvb/33/6

Published by the American Vacuum Society

\section{ARTICLES YOU MAY BE INTERESTED IN}

Displacement Talbot lithography nanopatterned microsieve array for directional neuronal network formation in brain-on-chip

Journal of Vacuum Science \& Technology B 34, 06 KI02 (2016); https://doi.org/10.1116/1.4961591

Recreating blood-brain barrier physiology and structure on chip: A novel neurovascular microfluidic bioreactor Biomicrofluidics 9, 054124 (2015); https://doi.org/10.1063/1.4934713

Passive pumping for the parallel trapping of single neurons onto a microsieve electrode array Journal of Vacuum Science \& Technology B 35, 06GA01 (2017); https://doi.org/10.1116/1.4991827

Hydrogel/poly-dimethylsiloxane hybrid bioreactor facilitating 3D cell culturing Journal of Vacuum Science \& Technology B 31, 06F903 (2013); https://doi.org/10.1116/1.4831762

Nanoscaffold's stiffness affects primary cortical cell network formation Journal of Vacuum Science \& Technology B 32, 06FD03 (2014); https://doi.org/10.1116/1.4900420

A zero-flow microfluidics for long-term cell culture and detection AIP Advances 5, 041310 (2015); https://doi.org/10.1063/1.4904071

\section{AVS Quantum Science}




\title{
Advances in 3D neuronal cell culture
}

\author{
Jean-Philippe Frimat \\ Department of Mechanical Engineering, Microsystems Group and ICMS Institute for Complex Molecular \\ Systems, Eindhoven University of Technology, 5612 AZ Eindhoven, The Netherlands
}

Sijia Xie

Mesoscale Chemical Systems, MESA+ Institute for Nanotechnology, University of Twente, 7500 AE Enschede, The Netherlands

Alex Bastiaens

Department of Mechanical Engineering, Microsystems Group and ICMS Institute for Complex Molecular Systems, Eindhoven University of Technology, 5612 AZ Eindhoven, The Netherlands

Bart Schurink

Mesoscale Chemical Systems, MESA+ Institute for Nanotechnology, University of Twente, 7500 AE Enschede, The Netherlands

Floor Wolbers ${ }^{\text {a) }}$ and Jaap den Toonder

Department of Mechanical Engineering, Microsystems Group and ICMS Institute for Complex Molecular Systems, Eindhoven University of Technology, 5612 AZ Eindhoven, The Netherlands

Regina Luttge ${ }^{\text {b) }}$

Department of Mechanical Engineering, Microsystems Group and ICMS Institute for Complex Molecular Systems, Eindhoven University of Technology, 5612 AZ Eindhoven, The Netherlands and Mesoscale Chemical Systems, MESA + Institute for Nanotechnology, University of Twente, 7500 AE Enschede, The Netherlands

(Received 26 June 2015; accepted 10 September 2015; published 24 September 2015)

In this contribution, the authors present our advances in three-dimensional (3D) neuronal cell culture platform technology contributing to controlled environments for microtissue engineering and analysis of cellular physiological and pathological responses. First, a micromachined silicon sieving structure is presented as key parameter for a modified version of a planar tissue culture, allowing seeding of single neurons in pyramidal shaped pores by a hydrodynamic sieve flow. Second, a nanogroove-hydrogel interface is presented as a more biomimetic in vivo representation of neuronal tissues, where 3D culturing is required to reproduce the layered tissue organization, which is observed in the microenvironment of the brain. To further our understanding of uniquely nanopatterned interfaces, the authors evaluated 3D neuronal outgrowth into Matrigel atop of primary cortical cell (CTX) cultured on nanogrooves. The interface facilitates conformation of cell somas and aligned outgrowth in 3D with outgrowth alignment preserved in Matrigel up to $6 \mu \mathrm{m}$ above the nanogrooved substrate, which has a pattern height of just $108 \mathrm{~nm}$. Finally, with the view to incorporate these guided culture interfaces in our previously designed hybrid Polydimethylsiloxane bioreactor, the authors have also explored 3D cellular culture matrix as a variable in such systems. By analyzing the effect of different gel matrices (Matrigel, PuraMatrix, and collagen-I) on the neuron model cell line SH-SY5Y, the authors bring together the ability to guide neuronal growth in spatially standardized patterns and within a bioreactor potentially coupled to an array of single cells that could facilitate readout of such complex cultures by integration with existing technologies (e.g., microelectrode arrays). Various combinations of these novel techniques can be made and help to design experimental studies to investigate how changes in cell morphology translate to changes in function but also how changes in connectivity relate to changes in electrophysiology. These latest advancements will lead to the development of improved, highly organized in vitro assays to understand, mimic, and treat brain disorders. (c) 2015 Author(s). All article content, except where otherwise noted, is licensed under a Creative Commons Attribution 3.0 Unported License. [http://dx.doi.org/10.1116/1.4931636]

\section{INTRODUCTION}

The development of in vitro brainlike tissue constructs is important for the understanding of brain physiology. In vivo studies are slow, low throughput, complex, and not

\footnotetext{
${ }^{\text {a)} P r e s e n t ~ a d d r e s s: ~ A c a d e m i e ~ L i f e ~ S c i e n c e, ~ E n g i n e e r i n g, ~ a n d ~ D e s i g n, ~ S a x i o n ~}$ University of Applied Sciences, M.H. Tromplaan 28, 7500 KB Enschede, The Netherlands.

b)Electronic mail: R.Luttge@tue.nl
}

predictable. In addition, animal use could be dramatically reduced for drug screening studies if good in vitro models were engineered, as they are high-throughput, reproducible, and robust, as well as cost-effective considering today's demand in pharmacological developments. ${ }^{1}$ There is therefore a need for improving 3D neuronal cell cultures models to create in vivo like brain tissue constructs on a chip for a miniaturized analytical display to study brain development and complex brain cells interactions leading to diseases. ${ }^{2}$ 
Engineering brainlike tissue constructs on a chip however is challenging and requires multidisciplinary integration skills. The brain is a complex yet highly organized network of cells communicating chemically and electrically with each other in a very specific manner that enable its functionality. ${ }^{3}$ Neurological disorders and diseases arise when the brain cellular network is disturbed (i.e., by structural, biochemical, or electrical damage), which can lead to Alzheimer, Parkinson's, and epilepsy, to name a few. Growing neurons in 3D and in a spatially standardized fashion will ease the analysis of neurite connectivity (which is a hallmark neurodevelopmental end point indicator) and will lead to an easier method for relating changes in connectivity to electrophysiology. Micro- and nanofabrication can be used for the parallel isolation and observation of cellular processes. In the field of neurobiology, microfluidic corridors and interconnected compartments have been applied to the study of axon guidance $^{4}$ and neuronal regeneration processes. ${ }^{5-8}$ For the spatiotemporal investigation of electrophysiological function, an ultimate neurodevelopmental end point, microelectrode arrays (MEA) can be used. ${ }^{9-13}$ These have been coupled with microfluidic compartments for the aligned monitoring of neuronal network activity. ${ }^{14-16}$ Alternatively, cell patterning ${ }^{17-21}$ can be used to interface neurons with electrodes for probing potential propagation at defined locations through engineered neuronal architectures ${ }^{22,23}$ and even along single axons. ${ }^{24}$ Axonal and dendritic outgrowths (collectively termed neurites) are defining morphological characteristics of the differentiated neuronal phenotypes that are essential for neuronal connectivity and network function. These neurites are dependent on many factors to properly function, such as matrix stiffness, cell composition, and biochemical and physical cues. ${ }^{25}$ For example, stiffness and topography affects cell attachment and outgrowth direction. ${ }^{26-28}$ Topographical cues such as micro- or nanogrooves have been employed to direct nerve regeneration ${ }^{29}$ and outgrowth direction in 2D (Refs. 30 and 31) whereas matrix stiffness can impact the phenotype and genotype of neuronal cells. ${ }^{31,32}$ In addition, mechanically driven cell polarization in brain tissues and neurotherapeutic approaches using functionalized supermagnetic nanoparticles to potentially restore disordered neural circuits have also been investigated. ${ }^{33}$

In order to achieve such a brain on a chip construct (Fig. 1), three main challenges must be addressed: (1) facilitating 3D conformation of cell soma and neurite outgrowth ideally at the level of a single cell, (2) understanding the impact of nanogrooves on 3D cell alignment, and (3) selecting culture matrix materials best suited to act as a 3D scaffold structure to be applied by a simple pipetting action. We first describe a microfabricated sieving structure for the generation of a single cell seeding platform, which utilizes hydrodynamic capturing of neurons. Here, single neurons can be analyzed very easily in an ordered fashion. This also allows every seeding platform to harbor arrays where the spatial confinement of cells enables analysis of network connectivity in a reproducible manner and can be coupled to a MEA or bioreactors, ${ }^{34}$ to directly relate changes in neuronal

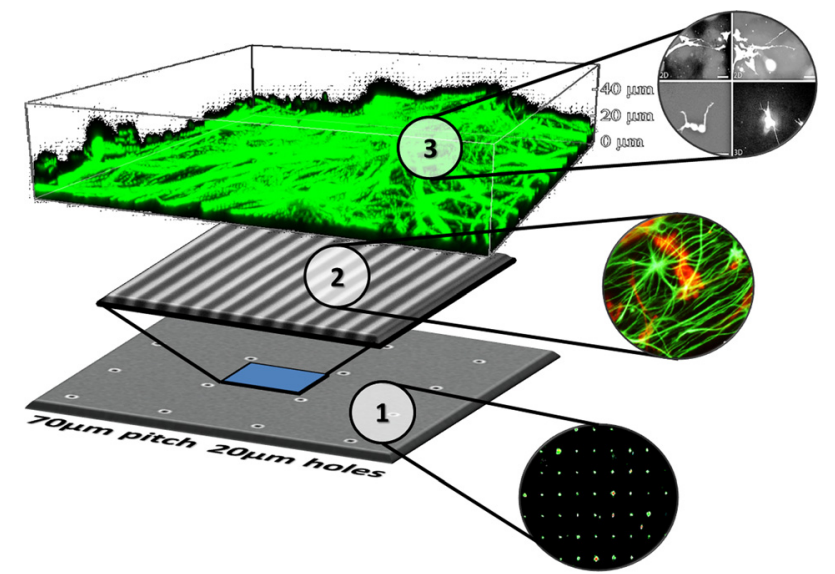

FIG. 1. (Color online) Concept of our brainlike tissue construct realized by advanced micro- and nanofabrication technology to study neuronal network behavior in 3D: Layer 1 represents the microsieve platform to array neurons. Layer 2 depicts the nanogrooved surfaces to direct and guide neurite and network formation in between the individual microsieves. Layer 3 shows cell cultured in hydrogels on top of the microsieve array and guiding nanogroove topographical features to enable cells to organize in a tissue like formation in $3 \mathrm{D}$.

connectivity to changes in electrophysiology. Second, in order to engineer brain like tissue constructs resembling the organized in vivo 3D architecture of the brain, 3D cell culturing and control over 2D and 3D cell growth must be achieved. State of the art MEMS technology is used to fabricate the microsieve array platform and is compatible with postprocessing fabrication to enable the inclusion of nanoimprinting lithography nanostructuring in between the pores of the sieve to control and guide the neurite outgrowth direction. Here, the effect of topographical surface cues (i.e., nanogrooves) on 3D cell alignment was investigated to elucidate the impact of surface cues for 3D cell culturing. Finally, since the stiffness of artificial substrates or scaffolds plays an important role in cell culturing, we have documented the behavior of neuron cells in three different cell matrices (biocompatible hydrogels) with stiffness comparable to the brain tissue, 300-500 Pa to further our understanding of $3 \mathrm{D}$ cell culturing. Put together, these advances will lead to the construction of a fully functional 3D neuronal tissue resembling the brain microarchitecture where cells can be arrayed, hence manipulated, and 3D connectivity can be tailored to mimic specific pathological scenarios.

\section{EXPERIMENT}

\section{A. Single cell seeding in 3D pores}

A sieve-structure is developed enabling hydrodynamic capturing of single cells in micron-sized pyramidal pores. This sieving-structure with a surface area of several square millimeters, featuring highly uniform pores and apertures, are fabricated by means of corner-lithography and wet chemical etching in $\{100\}$-silicon. ${ }^{35}$

Before seeding the cells into these pyramidal pores, the sieve-structure is accommodated with a Polydimethylsiloxane (PDMS) (Dow corning, Sylgard 184) reservoir (top side) and a PDMS slab (back side) by means of which the seeding 
platform is connected to a syringe. Rat cortical cells, at a density of 1000 cells in $10 \mu \mathrm{l} \mathrm{R} 12$ enriched culture medium, ${ }^{36}$ are brought into the reservoir and suction with the syringe facilitates a flow across the sieving structures, thereby positioning each of the cells into a pyramidal pore. After seeding, culture medium is added in the reservoir and refreshed every other day. After 14 days in vitro (DIV), the cells are fixed, dried and imaged by SEM (JEOL JSM 5610). A LIVE/DEAD ${ }^{\circledR}$ assay (Sigma) was performed to verify cell viability.

\section{B. Cell fixation and preparation for SEM}

The sieving structures with cells are fixed with $4 \%$ formaldehyde in $0.1 \mathrm{M}$ phosphate buffer for $30 \mathrm{~min}$. After fixation, the sieving structures are rinsed several times with phosphate buffered saline for $15 \mathrm{~min}$ and dehydrated through $70 \%, 80 \%, 90 \%$, and $100 \%$ ethyl alcohol for $15 \mathrm{~min}$ each. Chemical drying is then performed by 2 parts $100 \%$ ethyl alcohol/1 part hexamethyldisilazane for $15 \mathrm{~min}, 1$ part $100 \%$ ethyl alcohol/2 parts HDMS for $15 \mathrm{~min}$, then two changes for $15 \mathrm{~min}$ each with $100 \%$ HDMS. Finally, the HDMS is evaporated at room temperature in air-dry conditions overnight.

\section{Nanoscaffold fabrication}

The PDMS (Dow corning, Sylgard 184) nanoscaffolds were fabricated by soft lithography with a template of resist scaffolds formed by jet and flash imprint lithography $\left(\mathrm{J}-\mathrm{FIL}^{\mathrm{TM}}\right)$. Materials and the fabrication process details are described in our previous work. ${ }^{30,31}$ In brief, the patterning process contains the following steps: the scaffold was first fabricated by dispensing and imprinting the resist using the Imprio55 equipment (Molecular Imprints, Inc., USA) with a quartz stamp containing nanogrooved features on a silicon wafer precoated with bottom antireflective coating layer. Subsequently, the resist scaffold was copied by spin coating a PDMS layer with $100 \mu \mathrm{m}$ thickness and curing the PDMS at $80{ }^{\circ} \mathrm{C}$ for $30 \mathrm{~min}$. The PDMS copy with reversed structures was then peeled from the resist template and coated with PEI (polyethyleneimine) before using it for cell culturing.

\section{Cell culture and staining}

The primary cortical cells were isolated from a new born rat's cortex and were applied on top of the PEI coated nanoscaffolds in a standard 24 well culturing plate (CorningVR CostarVR), at a density of $4000-4500$ cells/ $\mu$ l. The CTX cells were seeded on the nanogrooved PDMS surfaces and allowed to stabilize and grow for $24 \mathrm{~h}$. A layer of Matrigel (BD Matrigel ${ }^{\mathrm{TM}}$ Basement Membrane Matrix) was then added on top and gelated by heat at $37^{\circ} \mathrm{C}$ inside an incubator with $5 \% \mathrm{CO}_{2}$. R12H media ${ }^{36}$ with penicillin/streptomycin antibiotics was periodically exchanged every 2 days, and the CTX cells were allowed to grow within the Matrigel matrix at $37^{\circ} \mathrm{C}$ and $5 \% \mathrm{CO}_{2}$ for 12 days until analysis. To study the behavior of astrocyte in the primary cortical cell culture, we performed immunostaining using astrocyte specific antiGlial Fibrillary Acidic Protein antibody (goat; Sigma, SAB2500462; 1:200) as primary antibody, and antigoat IgG
(HpL), $\mathrm{CF}^{\mathrm{TM}}$ 488A (donkey; Sigma Aldrich, SAB4600032; 1:200) as the secondary antibody. The staining protocol followed the standard protocol by Yale Center for high throughput cell biology. ${ }^{37}$ The primary cultures were stained with immunofluorescence (see above) and imaged through a depth-stack scanning with confocal microscopy (Nikon) every $300 \mathrm{~nm}$. Alignment was visually assessed by fluorescence and quantitatively measured.

\section{E. Matrix preparation and analysis}

To document the behavior of neuronal cells in response to different extracellular matrices, three types of gel matrices were tested in Ibidi $\mu$-Slides (Ibidi GmbH). SH-SY5Y neuroblastoma cell line (Sigma Aldrich) was used to characterize neuronal performance inside the different gel matrices. All reagents were purchased from Sigma Aldrich unless stated otherwise. Cells were cultured following the manufacturer protocol. Briefly cells were cultured in a 1:1 ratio mixture of Dulbecco's Modified Eagle Medium and F-12 medium supplemented with $10 \%$ Fetal Bovine Serum and 1\% pen-strep at $37^{\circ} \mathrm{C}$ in a $5 \% \mathrm{CO}_{2}$ environment. SH-SY5Y cell suspensions of 20000 cells $/ \mathrm{ml}$ were mixed in a 1:1 ratio to create 10000 cells $/ \mathrm{ml}$ samples with either growth factor reduced Matrigel (BD Matrigel ${ }^{\mathrm{TM}}$ Growth Factor Reduced Basement Membrane Matrix), $1.5 \mathrm{mg} / \mathrm{ml}$ collagen-I or $0.5 \% \mathrm{w} / \mathrm{v}$ PuraMatrix (BD PuraMatrix ${ }^{\mathrm{TM}}$ peptide hydrogel). Gelation for each of the matrices was done as per manufacturer protocol, where Matrigel was warmed up to $37^{\circ} \mathrm{C}$, collagen-I was brought to neutral $p \mathrm{H}$ and PuraMatrix gelled by adding medium. As a control, cells were also seeded at 10000 cells $/ \mathrm{ml}$ in $\mu$-slide (Ibidi) wells without any biogel matrix (2D). For differentiation into the neuronal lineage, cells were cultured 3 days in medium with added $10 \mu \mathrm{M}$ transretinoic acid (Sigma) within the gels, following which the performance was assessed over a period of 9 days (neurite outgrowth and cell soma size). At days 2, 7, and 9, the samples were fixed and stained using $0.1 \mathrm{mg} / \mathrm{ml}$ propidium iodide. For cells in Puramatrix, the samples were fixed and stained on days 4 and 8. Height-stack scans were made using confocal microscopy (Zeiss LSM 510) and assessed for cell soma size (topdown viewed largest diameter) and neurite outgrowth length.

\section{RESULTS AND DISCUSSION}

\section{A. Single cell seeding in 3D pores}

A sieve-structure is developed enabling hydrodynamic capturing of cells in pyramidal pores [Fig. 2(a)]. The silicon sieve is designed as an array, which contains $9003 \mathrm{D}$ pyramidal shaped pores with a square top opening of $20 \mu \mathrm{m}$ and a square aperture size at the bottom of the sieve of $3 \mu \mathrm{m}$ [Fig. 2(b)]. The pores are evenly distributed in a circular area of a radius of $1.2 \mathrm{~mm}$ with a pitch of $70 \mu \mathrm{m}$.

After 14 days in vitro (DIV), the cells in the sieving structures are stained to verify their viability and afterward their morphology is studied with SEM. The viability of the captured neurons, verified by the LIVE/DEAD assay (see supplementary Fig. $1^{38}$ ), is well within the $70 \%$ which we experienced from previous studies. ${ }^{30,34}$ This indicates that 


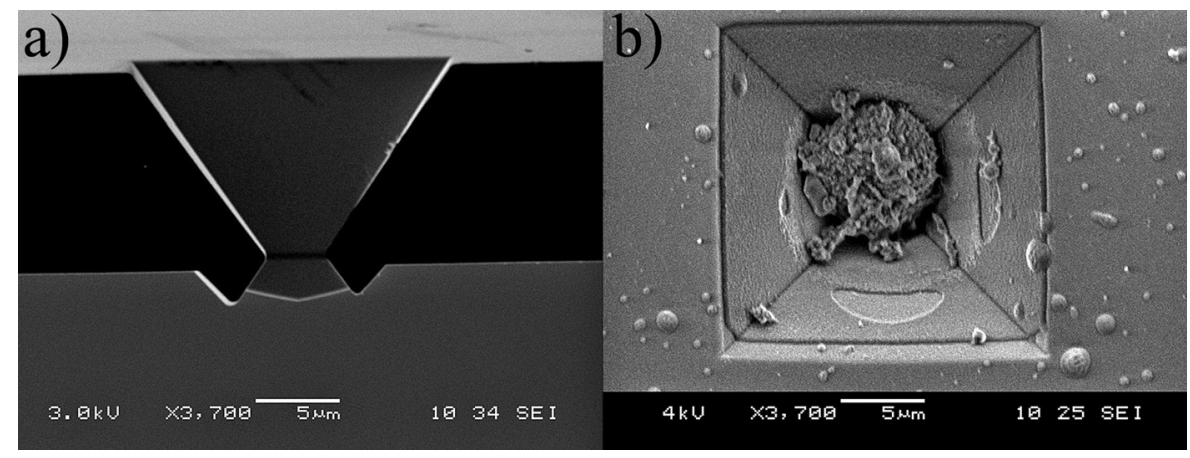

FIG. 2. (a) Cross-sectional SEM-image of one pore of the microfabricated silicon sieving structure, showing a single pyramidal shaped pore with base length of $20 \mu \mathrm{m}$ and an aperture of $3.2 \mu \mathrm{m}$. (b) SEM-image of the pyramidal shaped pore containing an adhered neuronal cell isolated from the cortex of newborn rats, yielding a round morphology similarly to culture in biogels (14 DIV). The hydrodynamic capturing of cells is highly efficient, as the number of occupied pores by neurons is over $80 \%$.

the materials of the sieving structure which are in contact with the cells and the seeding procedure have no negative influence on the cell viability. SEM analysis shows neurons, with a round morphology, adhered to the bottom part of the pyramidal pore. The round morphology of the neuron is the result of the 3D environment, providing multiple adhesion points in the pyramidal shaped pore. The hydrodynamic capturing of cells is highly efficient, as the number of occupied pores by neurons is over $80 \%$. The absence of large cell protrusions can be explained by the relative large distance $(70 \mu \mathrm{m})$ between each neuron and the lack of supporting cells in this setup. The final single cell seeding platform can be interfaced with our previously presented hybrid bioreactor. ${ }^{34}$

\section{B. Impact of surface nanogrooves on 3D cell alignment}

We previously reported that astrocytes from primary CTX cells can sense and orient themselves to nanogrooved patterned substrates. In addition, we showed that the level of alignment was similar for both hard (Silicon, GPa range) and soft (PDMS, kPa-MPa range) patterned surfaces, respectively, $85 \%$ and $90 \%$ alignment, indicating that topography not stiffness plays a crucial role in outgrowth alignment. ${ }^{30,31}$ In an effort to elucidate the impact surface nanogrooves may have on 3D cell culturing, we have cultured the CTX cells on the nanogrooved PDMS substrates and added Matrigel on top, a biogel serving as a biomimetic extracellular 3D scaffold. Following 12 days in culture, the alignment at different heights was recorded by confocal microscopy. The "aligned outgrowth" is defined as a deviation of the direction of the grooves within an angle of less than $30^{\circ}(\mathrm{n}=3)$. We demonstrate that astrocyte alignment can still be observed at a height of $6 \mu \mathrm{m}$ from the nanogrooves, indicating that cells keep their alignment when migrating upwards into the Matrigel scaffold (Fig. 3 and supplementary Fig. 2) ${ }^{38}$ and that nanoscale surface features can still have an influence at the micrometer scale. This indicates that layered topographical cues are critical for the creation of organized 3D cell cultures with nanogrooved interfaces.

\section{Neuronal cells inside 3D matrices}

The end goal of this project is to interface 3D neuronal cell cultures within a bioreactor ${ }^{34}$ to understand and control the relationship between connectivity and electrophysiology in brain like tissue constructs. Therefore, the behavior of the neuronal cells must be documented in a 3D matrix. We chose three different matrices: collagen-I, Matrigel, and PuraMatrix. These matrices can be gelated to provide a 3D environment where the cells can sit and grow. In addition, these biocompatible gels have stiffness related to the brain, between 200 and $500 \mathrm{~Pa}$, making them suitable for mimicking the in vivo brain's ECM (Extra Cellular Matrix). The neuroblastoma cell line SHSY5Y was chosen as the neuron cell line candidate for assessing the effect of culturing cells in 3D matrices. Here, we show that following 8 and 9 days in culture, neurite outgrowth was similar in all samples $(2 \mathrm{D}=50 \pm 23$ and $3 \mathrm{D}=40 \pm 27 \mu \mathrm{m})$ $(\mathrm{n}=3)$, indicating that $3 \mathrm{D}$ culturing and matrix composition did not affect the ability of the neurites to grow (comparable to 2D lengths) [Fig. 4(a)]. However, a major difference was observed when comparing cell soma sizes between 2D and 3D samples [Fig. 4(b)]. On 2D surfaces, the cells are allowed to spread and as a consequence their overall morphology is large and flat $(44 \pm 7 \mu \mathrm{m})(\mathrm{n}=3)$. In 3D conformation, the cells are not allowed to spread, and as a consequence, their overall morphology is small and round $(19 \pm 5 \mu \mathrm{m})(\mathrm{n}=3)$ (supplementary Fig. $3^{38}$ ). Similarly, these changes in cell soma sizes were also observed for the cortical astrocytes grown on soft PDMS surfaces, ${ }^{30,31}$ indicating that although material stiffness does not affect outgrowth length and alignment, stiffness does affect the cell morphology and therefore potentially its function. Indeed, previous reports have identified genetic variations in correlation with morphological changes in the SH-SY5Y cell line grown in 3D compared to 2D. In 3D, genes encoding cytoskeletal-associated proteins such as actin filament $\alpha 2$ capping protein and signal transduction factors such as midkine were upregulated. Genes encoding the cytoskeletal proteins filamin $\mathrm{A}$, actinin $1 \alpha 1$, and talin 1 and genes encoding $\mathrm{ECM}$ molecules such as fibronectin 1, collagen III $\alpha 1$, and versican were all downregulated in $3 \mathrm{D} .{ }^{32}$ These analyses were performed in both Matrigel and collagen-I gels and showed the same trend, indicating that the cell is able to sense its $3 \mathrm{D}$ 


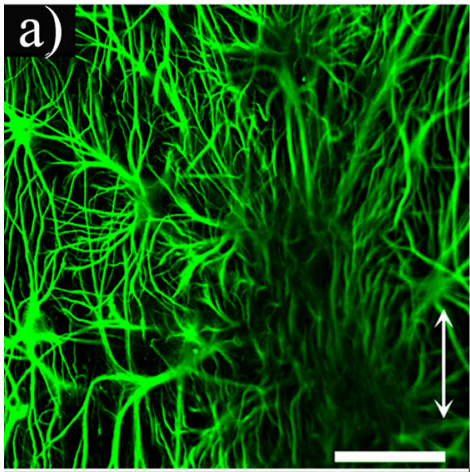

\section{c) Outgrowth alignment in 3D cultures}
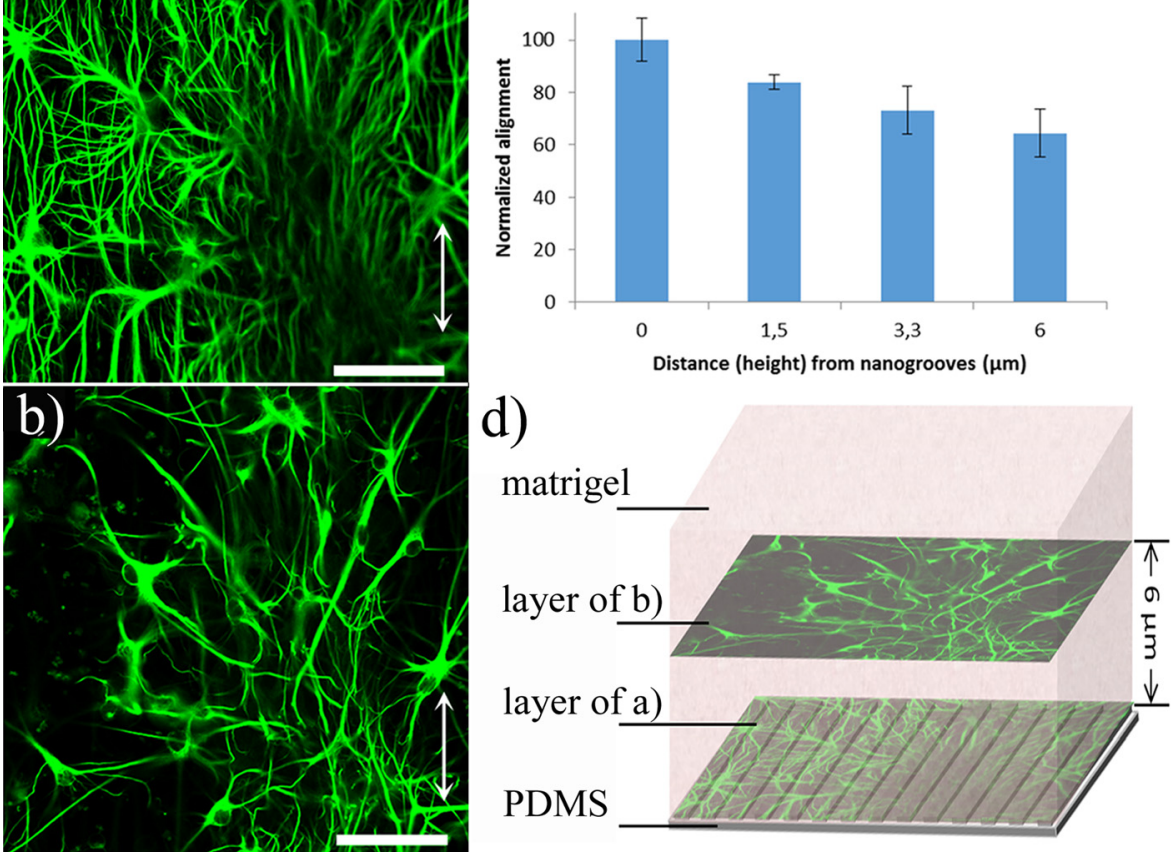

FIG. 3. (Color online) Example of CTX primary astrocytes aligned to the nanogrooves at (a) $0 \mu \mathrm{m}$ and at (b) $6 \mu \mathrm{m}$ is shown. The white arrows indicate the parallel direction of the nanogrooves. Scale bars $=50 \mu \mathrm{m}$. (c) Normalized outgrowth alignment against 3D culture height showing that $6 \mu \mathrm{m}$ from the nanogrooves, $65 \%$ of alignment is still observable. The "aligned outgrowth" is defined as a deviation of the grooves direction within an angle of less than $30^{\circ}$ $(n=3)$. (d) Schematic drawing of the spatial positions of the scaffolds and the cells. The images of cells in 3D culture were taken from top view.

environment and adopt a more in vivo like morphology as a consequence. ${ }^{39}$ In addition, Choi et al. ${ }^{40}$ also used quantitative Polymerase Chain Reaction to demonstrate that neuron maturation is promoted in $3 \mathrm{D}$ cultures and tau expression is increased, which is essential for reconstituting tauopathy, an

a)

Neurite length: 2D vs 3D

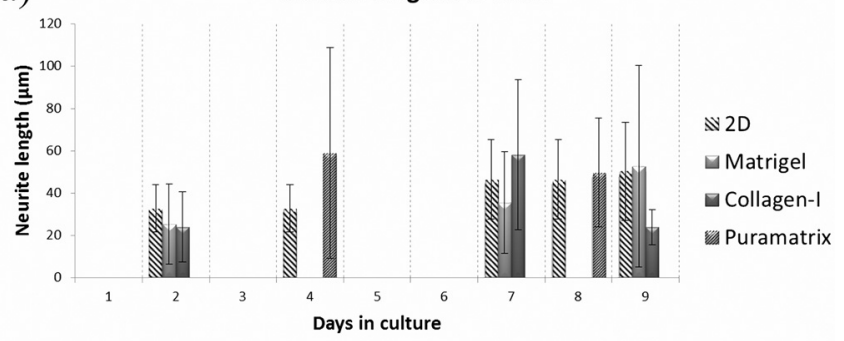

b)

Cell soma size: 2D vs 3D

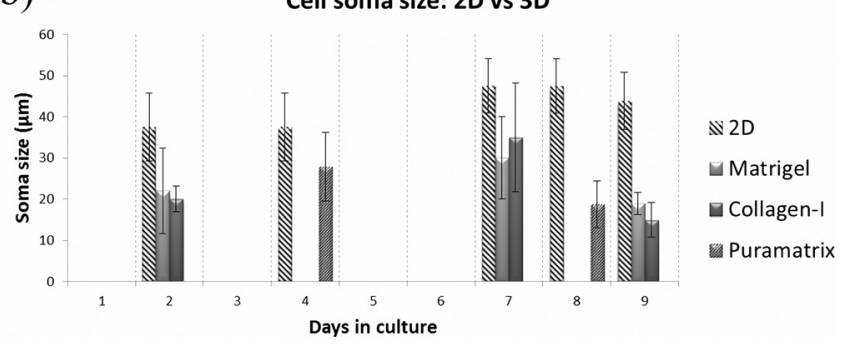

FIG. 4. Comparison of SH-SY5Y differentiated cells on flat surfaces (2D, polystyrene) and inside different biogels (3D = Matrigel, collagen-I and PuraMatrix). (a) Neurite outgrowth length measurements are comparable after 9 days in culture between $2 \mathrm{D}$ and 3D cultures, averaging at $50 \pm 23 \mu \mathrm{m}$ for $2 \mathrm{D}$ and $40 \pm 27 \mu \mathrm{m}$ for $3 \mathrm{D}$ in length $(\mathrm{n}=3)$. (b) SH-SY5Y cell size decreases from $44 \pm 7 \mu \mathrm{m}$ for $2 \mathrm{D}$ flat surfaces to $19 \pm 5 \mu \mathrm{m}(\mathrm{n}=3)$ inside 3D biogels after a period of 9 days. important feature in Alzheimer disease. It is however important to notice that $3 \mathrm{D}$ culturing results in the formation of highly disorganized cellular networks that make morphological measurements such as neurite outgrowth length challenging. The slightest variation in cell concentration and cell positioning within the sample translates into variations in those measurements, as evident from the error bars in Fig. 4. If neurons are close to each other, their connecting neurite outgrowth will be shorter than if the neurons were standing further apart. Hence, spatial standardization or cell patterning could be hugely beneficial to standardize the distances between neurons (standardizing outgrowth lengths) and enabling reproducible measurements to be recorded and compared. ${ }^{21}$ Since relating connectivity to electrophysiology is the focus of the project, control over the level of connectivity inside the 3D sample is as important as controlling the connectivity at the interface between neurons and electrodes. In our next efforts, we will investigate how cells can be organized at the $3 \mathrm{D}$ level.

\section{CONCLUSIONS}

We have developed a novel single cellular sieving structure that allows hydrodynamic arraying of single neurons generating a spatially standardized analytical display for neuron studies. We have shown that 2D surface nanoscale cues can affect 3D cell cultures where PDMS engineered guiding nanogrooves with heights of $108 \mathrm{~nm}$ impart neurite outgrowth alignment up to $6 \mu \mathrm{m}$ inside the biogel scaffolds (height). We have also demonstrated that 3D cell culturing of SH-SY5Y differentiated neurons inside biogel scaffolds leads to changes 
in cell morphology which resemble the in vivo neuron morphology (round in shape, smaller in size $\pm 20 \mu \mathrm{m}$ ). In summary, we designed and realized spatial control features for the organized formation of neuronal networks.

\section{ACKNOWLEDGMENTS}

This project was financially supported by the ERC, Grant No. 280281 (MESOTAS). The authors sincerely thank B. Vratzov (MESA+ Institute) for assisting in J-FIL, and B. Klomphaar and G. Hassink for providing dissociated cells (MIRA Institute). The authors also thank C. V. C. Bouten at the faculty of BioMedical Technology and Soft Tissue Biomechanics and Tissue Engineering group at the Technical University of Eindhoven, Netherlands, for equipment and laboratory access.

${ }^{1}$ A. D. van der Meer and A. van den Berg, Integr. Biol. 4, 461 (2012).

${ }^{2}$ M. A. Lancaster et al., Nature 501, 373 (2013).

${ }^{3}$ O. Sporns, Nat. Neurosci. 17, 652 (2014).

${ }^{4}$ N. D. Dinh et al., Lab Chip 13, 1402 (2013).

${ }^{5}$ A. M. Taylor, S. W. Rhee, C. H. Tu, D. H. Cribbs, C. W. Cotman, and N. L. Jeon, Langmuir 19, 1551 (2003).

${ }^{6}$ A. M. Taylor, M. Blurton-Jones, S. W. Rhee, D. H. Cribbs, C. W. Cotman, and N. L. Jeon, Nat. Methods 2, 599 (2005).

${ }^{7}$ Y.-T. Kim, K. Karthikeyan, S. Chirvi, and D. P. Dave, Lab Chip 9, 2576 (2009).

${ }^{8}$ D. K. Cullen, M. D. Tang-Schomer, L. A. Struzyna, A. R. Patel, V. E. Johnson, J. A. Wolf, and D. H. Smith, Tissue Eng. Part A 18, 2280 (2012).

${ }^{9}$ C. A. Thomas, P. A. Springer, G. E. Loeb, Y. Berwald-Netter, and L. M. Okun, Exp. Cell Res. 74, 61 (1972).

${ }^{10}$ E. W. Keefer, A. Gramowski, D. A. Stenger, J. J. Pancrazio, and G. W. Gross, Biosens. Bioelectron. 16, 513 (2001).

${ }^{11}$ A. Tscherter, M. O. Heuschkel, P. Renaud, and J. Streit, Eur. J. Neurosci. 14, 179 (2001).

${ }^{12}$ E. van Vliet, L. Stoppini, M. Balestrino, C. Eskes, C. Griesinger, T. Sobanski, M. Whelan, T. Hartung, and S. Coecke, Neurotoxicology 28, 1136 (2007).

${ }^{13}$ J. Ban, P. Bonifazi, G. Pinato, F. D. Broccard, L. Studer, V. Torre, and M. E. Ruaro, Stem Cells 25, 738 (2007).

${ }^{14}$ I. Suzuki, Y. Sugio, Y. Jimbo, and K. Yasuda, Lab Chip 5, 241 (2005).

${ }^{15}$ S. K. Ravula, M. A. McClain, M. S. Wang, J. D. Glass, and A. B. Frazier, Lab Chip 6, 1530 (2006).
${ }^{16}$ F. Morin, N. Nishimura, L. Griscom, B. Le Pioufle, H. Fujita, Y. Takamura, and E. Tamiya, Biosens. Bioelectron. 21, 1093 (2006).

${ }^{17}$ M. Mrksich, L. E. Dike, J. Tien, D. E. Ingber, and G. M. Whitesides, Exp. Cell Res. 235, 305 (1997).

${ }^{18}$ H. Hardelauf et al., Lab Chip 11, 2763 (2011).

${ }^{19}$ A. Folch and M. Toner, Annu. Rev. Biomed. Eng. 2, 227 (2000).

${ }^{20}$ G. M. Whitesides, E. Ostuni, S. Takayama, X. Jiang, and D. E. Ingber, Annu. Rev. Biomed. Eng. 3, 335 (2001).

${ }^{21}$ J.-P. Frimat et al., Lab Chip 10, 701 (2010).

${ }^{22}$ E. V. Romanova, K. A. Fosser, S. S. Rubakhin, R. G. Nuzzo, and J. V. Sweedler, FASEB J. 18, 1267 (2004).

${ }^{23}$ Y. Mourzina, D. Kaliaguine, P. Schulte, and A. Offenhausser, Anal. Chim. Acta 575, 281 (2006).

${ }^{24}$ F. Patolsky, B. P. Timko, G. Yu, Y. Fang, A. B. Greytak, G. Zheng, and C. M. Lieber, Science 313, 1100 (2006).

${ }^{25}$ K. Franze, P. A. Janmey, and J. Guck, Annu. Rev. Biomed. Eng. 15, 227 (2013).

${ }^{26}$ P. C. Georges, W. J. Miller, D. F. Meaney, E. S. Sawyer, and P. A. Janmey, Biophys. J. 90, 3012 (2006).

${ }^{27}$ E. S. Ereifej, H. W. Matthew, G. Newaz, A. Mukhopadhyay, G. Auner, I. Salakhutdinov, and P. J. VandeVord, J. Biomed. Mater. Res. Part A 101, 1743 (2013)

${ }^{28}$ L. M. Y. Yu, N. D. Leipzig, and M. S. Shoichet, Mater. Today 11, 36 (2008).

${ }^{29}$ E. A. Bremus-Koebberling, S. Beckemper, B. Koch, and A. Gillner, J. Laser Appl. 24, 042013 (2012).

${ }^{30}$ S. Xie and R. Luttge, Microelectron. Eng. 124, 30 (2014).

${ }^{31}$ S. Xie, B. Schurink, F. Wolbers, R. Luttge, and G. Hassink, J. Vac. Sci. Technol. B 32, 06FD03 (2014).

${ }^{32}$ G. N. Li, L. L. Livi, C. M. Gourd, E. S. Deweerd, and D. Hoffman-Kim, Tissue Eng. 13, 1035 (2007).

${ }^{33}$ A. Kunze, P. Tseng, C. Godzich, C. Murray, A. Caputo, F. E. Schweizer, and D. Di Carlo, ACS Nano 9, 3664 (2015).

${ }^{34}$ B. Schurink and R. Luttge, J. Vac. Sci. Technol. B 31, 06 F903 (2013).

${ }^{35}$ B. Schurink, J. W. Berenschot, R. M. Tiggelaar, and R. Luttge, Microelectron. Eng. 144, 12 (2015).

${ }^{36}$ H. J. Romijn, F. van Huizen, and P. S. Wolters, Neurosci. Biobehav. Rev. 8, 301 (1984).

37"“SIGMA-ALDRICH," http://www.sigmaaldrich.com/content/dam/sigmaaldrich/docs/Sigma/General_Information/1/yale-if-procedure.pdf

${ }^{38}$ See supplementary material at http://dx.doi.org/10.1116/1.4931636 for live dead staining on the micro sieve device, $\mathrm{Z}$ stacking of rat cortical astrocytes cultured on a nanogrooved surface, and a comparison of $\mathrm{SH}$ SY5Y cells growing on a flat 2D surface and SH-SY5Y cells growing in 3D inside Matrigel.

${ }^{39}$ F. Appaix et al., PLoS One 7, e35169 (2012).

${ }^{40}$ S. H. Choi et al., Nature 515, 274 (2014). 